\title{
Involvement of hepatic stellate cell cytoglobin in acute hepatocyte damage through the regulation of CYP2E1-mediated xenobiotic metabolism
}

\author{
Yuga Teranishi ${ }^{1,5}$, Tsutomu Matsubara ${ }^{2,5}$, Kristopher W Krausz ${ }^{3}$, Thi $\Pi$ Le, Frank J Gonzalez ${ }^{3}$, \\ Katsutoshi Yoshizato ${ }^{1,4}$, Kazuo Ikeda ${ }^{2}$ and Norifumi Kawada ${ }^{1}$
}

Oxygen $\left(\mathrm{O}_{2}\right)$ is required for cytochrome P450 (CYP)-dependent drug metabolism. Cytoglobin (CYGB) is a unique globin expressed exclusively in hepatic stellate cells (HSCs). However, its role in $\mathrm{O}_{2}$-dependent metabolism in neighboring hepatocytes remains unknown. This study provides evidence that CYGB in HSCs is involved in acetaminophen ( $N$-acetyl-p-aminophenol; APAP)-induced hepatotoxicity. Serum alanine aminotransferase levels were higher in wild-type mice than in Cygb-null mice. Wild-type mice exhibited more severe hepatocyte necrosis around the central vein area compared with Cygb-null mice, thus indicating that CYGB deficiency protects against APAP-induced liver damage. Although no difference in the hepatic expression of CYP2E1, a key enzyme involved in APAP toxicity, was observed between wild-type and Cygb-null mice, the serum levels of the APAP metabolites cysteinyl-APAP and $N$-acetyl-cysteinylAPAP were decreased in Cygb-null mice, suggesting reduced APAP metabolism in the livers of Cygb-null mice. In primary cultures, APAP-induced hepatocyte damage was increased by co-culturing with wild-type HSCs but not with Cygb-null HSCs. In addition, cell damage was markedly alleviated under low $\mathrm{O}_{2}$ condition $\left(5 \% \mathrm{O}_{2}\right)$, suggesting the requirement of $\mathrm{O}_{2}$ for APAP toxicity. Carbon tetrachloride-induced liver injury (CYP2E1-dependent), but not lipopolysaccharide/ D-galactosamine-induced injury (CYP2E1-independent), was similarly alleviated in Cygb-null mice. Considering the function of $\mathrm{CYGB}$ as $\mathrm{O}_{2}$ carrier, these results strongly support the hypothesis that HSCs are involved in the CYP2E1-mediated xenobiotic activation by augmenting $\mathrm{O}_{2}$ supply to hepatocytes. In conclusion, CYGB in HSCs contributes to the CYP-mediated metabolism of xenobiotics in hepatocytes by supplying $\mathrm{O}_{2}$ for enzymatic oxidation. Laboratory Investigation (2015) 95, 515-524; doi:10.1038/labinvest.2015.29; published online 16 February 2015

The metabolism of xenobiotics, including clinical drugs, occurs primarily in the liver. Most xenobiotic detoxification metabolism involves cytochrome P450 (CYP) enzymes, which are predominantly expressed in the liver, ${ }^{1}$ and these processes occasionally result in acute hepatocyte damage. For instance, acetaminophen ( $N$-acetyl- $p$-aminophenol; APAP) and carbon tetrachloride $\left(\mathrm{CCl}_{4}\right)$ are metabolized by CYP to generate toxic intermediates.

APAP is commonly used as an antipyretic and analgesic drug. CYP-mediated APAP metabolism is known to generate $\mathrm{N}$-acetyl-p-benzoquinone imine (NAPQI), which binds to cellular macromolecules and initiates hepatocyte damage if present at high concentrations under glutathione (GSH) depletion. $^{2}$ CYP1A2, CYP2A6, CYP2E1, and CYP3A have been identified as enzymes that generate NAPQI from APAP. ${ }^{3}$ Among these CYPs, CYP2E1 has the lowest $K_{\mathrm{m}}$ for APAP, and the CYP2E1-dependent metabolism of APAP can result in hepatotoxicity. ${ }^{4}$ CYP2E1 is a monooxygenase that requires molecular oxygen $\left(\mathrm{O}_{2}\right)$ for its enzymatic activity. A recent study reported that the APAP-induced damage to primary mouse hepatocytes was reduced under a low $\mathrm{O}_{2}$ concentration $\left(5 \% \mathrm{O}_{2}\right)$ compared with normoxic conditions $(20 \%$ $\left.\mathrm{O}_{2}\right) .^{5}$ Thus, APAP-induced acute liver injury may be dependent on hepatic $\mathrm{O}_{2}$ levels.

The aerobic metabolism of mammals relies on respiratory proteins that function in the transport and storage of $\mathrm{O}_{2}$.

\footnotetext{
'Department of Hepatology, Graduate School of Medicine, Osaka City University, Osaka, Japan; ${ }^{2}$ Department of Anatomy and Regenerative Biology, Graduate School of Medicine, Osaka City University, Osaka, Japan; ${ }^{3}$ Laboratory of Metabolism, Center for Cancer Research, National Cancer Institute, National Institutes of Health, Bethesda, MD, USA and ${ }^{4}$ Phoenixbio Co., Ltd, Hiroshima, Japan

Correspondence: Professor N Kawada, MD, PhD, Department of Hepatology, Graduate School of Medicine, Osaka City University, 1-4-3, Asahimachi, Abeno, Osaka 5458585, Japan. 
Four globin proteins are known to contribute to $\mathrm{O}_{2}$ homeostasis in mammals. ${ }^{6,7}$ Hemoglobin in erythrocytes has an important role in the transport of $\mathrm{O}_{2}$ from the bloodstream to the viscera. In the viscera, myoglobin, neuroglobin, and cytoglobin (CYGB) act as local $\mathrm{O}_{2}$-binding molecules that facilitate the intracellular diffusion of $\mathrm{O}_{2} \cdot{ }^{8}$ Notably, CYGB in mammals is expressed in various organs, including the liver, heart, brain, and the lung. CYGB is a hexacoordinated hemecontaining protein that is able to bind $\mathrm{O}_{2}$, nitric oxide, and carbon monoxide. ${ }^{9,10}$ CYGB expression increases in response to the duration and severity of hypoxia. ${ }^{11}$ Thus, CYGB is thought to be an $\mathrm{O}_{2}$ sensor for the local storage and transport of $\mathrm{O}_{2}$; however, its contribution to $\mathrm{O}_{2}$-dependent xenobiotic metabolism remains unclear.

CYGB is a unique globin expressed exclusively in hepatic stellate cells (HSCs), which represent $\sim 5-8 \%$ of the cells in the liver. ${ }^{12}$ When liver injury occurs, HSCs undergo activation and acquire myofibroblastic characteristics, including increased collagen production. Such characteristic alterations of HSCs are believed to be strongly associated with hepatic fibrosis and the development of liver cirrhosis and cancer. In addition, interactions between hepatocytes and HSCs, as well as the involvement of HSCs in hepatic tissue growth and regeneration have been reported. ${ }^{13,14}$ However, the interplay between HSCs and hepatocytes in drug metabolism remains uncharacterized. Furthermore, the effect of CYGB in HSCs (HSC-CYGB) on APAP-induced acute liver injury has never been investigated.

In this study, the correlation between HSC-CYGB and xenobiotic-mediated hepatocyte injury was investigated in vivo and in vitro using $C y g b$-null mice. The results implied that a CYGB deficiency in HSCs can regulate hepatic $\mathrm{O}_{2}$ levels and can alleviate the acute liver injury induced by APAP and $\mathrm{CCl}_{4}$, by decelerating CYP2E1 metabolism in hepatocytes.

\section{MATERIALS AND METHODS Materials}

All reagents were obtained from Sigma-Aldrich (St Louis, MO, USA) or Wako Pure Chemical (Osaka, Japan), unless otherwise noted.

\section{Mouse Studies}

Eight- to 12-week-old male C57BL/6 mice were purchased from Japan SLC (Shizuoka, Japan). Cygb-null mice were generated in our laboratory as previously described. ${ }^{15}$ Cygb-null mice were compared with their wild-type littermates. All mice were cared for according to the guidelines approved by the Institutional Animal Care and Use Committee of Osaka City University, Osaka, Japan. Before APAP treatment, the mice were fasted for $16 \mathrm{~h}$. APAP dissolved in isotonic saline at $65^{\circ} \mathrm{C}$ was intraperitoneally (i.p.) injected $(300 \mathrm{mg} / \mathrm{kg})$ into mice, which were killed $6 \mathrm{~h}$ later. In the study of $\mathrm{CCl}_{4}$-induced acute liver injury, mice were i.p. injected with $\mathrm{CCl}_{4}(0.5 \mathrm{mg} / \mathrm{kg})$ suspended in corn oil, fasted, and killed $24 \mathrm{~h}$ after the injection. Another group of mice were administered D-galactosamine (D-GalN; $700 \mathrm{mg} / \mathrm{kg}$ ) and lipopolysaccharide (LPS; $5 \mu \mathrm{g} / \mathrm{kg}$, Escherichia coli 0111:B4) suspended in saline and were killed $6 \mathrm{~h}$ later. Blood and liver tissues were collected and stored at $-80{ }^{\circ} \mathrm{C}$ for subsequent analysis. For histopathological analysis, small pieces of the liver were fixed in $4 \%$ formaldehyde overnight and embedded in paraffin. The liver tissue was sectioned at $5-\mu \mathrm{m}$ thickness and stained with hematoxylin and eosin. Relative necrotic area (\%) was estimated by using Image J software.

\section{Isolation, Fractionation, and Culture of Liver Cells}

Hepatocytes and HSCs were isolated from male C57BL/6 mice as previously described. ${ }^{16} \operatorname{APAP}(2.5,5,10,20$, or $30 \mathrm{mM}$ ) was dissolved in William's Medium E (Gibco, Grand Island, NY) at $65^{\circ} \mathrm{C}$. Hepatocytes were plated in 12- or 24-well plates $\left(1 \times 10^{5}\right.$ cells $\left./ \mathrm{ml}\right)$ (Celltight C-1 Collagen-I, Sumitomo Bakelite, Tokyo, Japan) and were pre-incubated in William's Medium E supplemented with $10 \%$ fetal bovine serum (Gibco) and $0.1 \mu \mathrm{M}$ dexamethasone for $3 \mathrm{~h}$ before APAP treatment. For co-culture experiments, HSCs $\left(5 \times 10^{5}\right.$ cells/well) were plated in cell culture inserts (Transparent PET membrane, $1.0 \mu \mathrm{m}$ pore size; Corning, Corning, NY, USA) and incubated overnight in the William's Medium E before co-culture with hepatocytes. The cells were incubated for $16 \mathrm{~h}$ with APAP-containing media under a $5 \%$ or $20 \%$ $\mathrm{O}_{2}$ atmosphere. Conditioned media derived from the 16-h culture of wild-type or Cygb-null HSCs were also used.

\section{Measurement of ALT Activity and APAP Metabolites in Serum}

Serum alanine aminotransferase (ALT) activity was determined using a kinetic test (SRL, Tokyo, Japan). The levels of APAP and its metabolites in sera collected $2 \mathrm{~h}$ after APAP injection were quantified as previously reported. ${ }^{4}$

\section{Visualization of Hypoxia With Pimonidazole}

Mice were injected with APAP $(300 \mathrm{mg} / \mathrm{kg}$, i.p.), followed by the administration of pimonidazole hydrochloride $(60 \mathrm{mg} / \mathrm{kg}$, i.p.; Hypoxyprobe, Burlington, MA, USA) $1 \mathrm{~h}$ before being killed. Small liver pieces were fixed in $4 \%$ paraformaldehyde and embedded in paraffin. Paraffin sections $(5 \mu \mathrm{m})$ were prepared in the Central Laboratory of the Osaka City University Medical School. Pimonidazole was detected using a Hypoxyprobe-1 Plus Kit, according to the manufacturer's instructions. An anti-fluorescein antibody conjugated to Texas Red (Ab6654; Abcam, Cambridge, UK) was used to visualize the reaction.

\section{GSH Measurements}

Liver tissues $(100 \mathrm{mg})$ were homogenized in $1 \mathrm{ml}$ of ice-cold phosphate-buffered saline containing $1 \mathrm{mM}$ EDTA ( $\mathrm{pH}$ 7.5) and were centrifuged at $10500 \mathrm{~g}$ for $15 \mathrm{~min}$ at $4{ }^{\circ} \mathrm{C}$. The supernatants were collected and the GSH content in each 
sample was determined using a GSH quantification kit (Dojindo, Kumamoto, Japan).

\section{Quantitative Real-Time Reverse Transcription-PCR Analyses}

Total RNA was isolated using TRIzol reagent (Invitrogen, Carlsbad, CA). cDNA was generated with random hexamer primers (Invitrogen) and the SuperScript RT II enzyme (Invitrogen). Quantitative PCRs were performed using the ABI 5500 Real-Time PCR System (Applied Biosystems, Foster City, CA, USA). The primers used are listed in Supplementary Table 1. Relative expression levels were calculated using the comparative CT method. The expression levels were normalized to that of $18 \mathrm{~S}$ ribosomal RNA.

\section{Western Blotting}

Liver tissues were homogenized with RIPA buffer $(50 \mathrm{mM}$ Tris- $\mathrm{HCl} \mathrm{pH}$ 8.0, $150 \mathrm{mM} \mathrm{NaCl}, 1 \%$ Triton X-100, 1\% SDS) containing a protease inhibitor (Roche, Basel, Switzerland) and phosphatase inhibitors ( $1 \mathrm{mM}$ sodium fluoride, $1 \mathrm{mM}$ $\beta$-glycerol phosphate, and $1 \mathrm{mM}$ sodium vanadate) and were centrifuged at $10000 \mathrm{~g}$ for $10 \mathrm{~min}$. The supernatants $(25 \mu \mathrm{g}$ protein) were subjected to western blot analysis of CYP2E1. To obtain nuclear extracts, liver tissues were prepared using the NE-PER Nuclear and Cytoplasmic Extraction Reagents kit (Thermo Fisher Scientific, Waltham, MA), according to the manufacturer's protocol. The nuclear extracts $(20 \mu \mathrm{g}$ protein) were subjected to western blot analysis of HIF- $1 \alpha$. Primary hepatocytes, cultured under a hypoxic conditions $\left(1 \% \mathrm{O}_{2}\right)$, were used as positive control $(5 \mu \mathrm{g})$ for western blotting of HIF- $1 \alpha$. The samples were separated on SDS-polyacrylamide gels and transferred to polyvinylidene fluoride membranes using standard western blotting techniques. The membranes were incubated with an antibody against CYP2E1 (kindly provided by Dr Koichi Yoshinari (Tohoku University, Sendai, Japan)) at a dilution of 1:10 000 or with an antibody against HIF- $1 \alpha$ (NB100-449, Novus Biologicals, Littleton, CO) at a dilution of 1:1000. The CYP2E1 protein levels in the samples were normalized to those obtained with a glyceraldehyde-3-phosphate dehydrogenase antibody at a dilution of 1:10000 (MAB374; Millipore, Billerica, MA, USA). The expression of HIF- $1 \alpha$ was normalized to the signal obtained with Coomassie brilliant blue staining.

\section{Measurement of Hepatic CYP2E1 Activity In Vitro}

Liver tissues $(100 \mathrm{mg})$ were homogenized in $1 \mathrm{ml}$ of ice-cold $50 \mathrm{mM}$ potassium phosphate buffer ( $\mathrm{pH}$ 7.4). The homogenate was centrifuged at $9000 \mathrm{~g}$ for $20 \mathrm{~min}$. The supernatant was transferred to a new tube and centrifuged at $105000 \mathrm{~g}$ for $60 \mathrm{~min}$. The pellet was suspended with $50 \mathrm{mM}$ Tris- $\mathrm{HCl}$ (pH 7.4)/20\% glycerol. The suspension was subjected to CYP2E1 activity assay with vivid CYP2E1 blue screening kit (Invitrogen).

\section{Assessment of Cell Death and Viability in Primary-Cultured Mouse Hepatocytes}

Cell death was analyzed with $1 \mu \mathrm{g} / \mathrm{ml}$ propidium iodide (Dojindo), which stains nuclei only when the cellular plasma membranes become permeabilized, and with Hoechst 33342 (Dojindo). Cell viability was determined using an MTT assay. ${ }^{17}$ The cell viability (\%) of the test samples was calculated relative to that of the control sample.

\section{Statistical Analysis}

The statistical analysis was performed using Prism version 6.0 software (GraphPad Software, San Diego, CA, USA). A $P$-value $<0.05$ was considered to indicate a significant difference.

\section{RESULTS \\ Alleviation of APAP-Induced Liver Injury in Cygb-Null Mice}

A single injection of APAP (300 mg/kg, i.p.) into C57Bl/6 mice induced significant liver injury, which was accompanied by a marked increase in the serum ALT levels (Supplementary Figure S1A) and a moderate to marked centrilobular necrosis (Supplementary Figure S1B) 6-12 h after the injection. Thus, in the subsequent experiments, wild-type and Cygb-null mice were killed $6 \mathrm{~h}$ after APAP injection.

The increase in serum ALT levels was significantly attenuated in the Cygb-null mice receiving APAP: the ALT levels of the wild-type and Cygb-null mice were $13970 \pm 2370$ and $4699 \pm 1226 \mathrm{U} / \mathrm{l}$, respectively (Figure 1a). In accordance with these results, the histological area of hepatocyte necrosis was diminished in the Cygb-null mice (Figure 1b). Relative necrotic area was larger in wild-type $(47.3 \pm 11.0 \%)$ than in Cygb-null mice $(17.8 \pm 5.0 \%)$ as shown in Figurelc. The APAP-induced increase in the hepatic mRNA levels of chemokine $(\mathrm{C}-\mathrm{C}$ motif) ligand $2(\mathrm{Ccl} 2)$ in the $\mathrm{Cyg} b$-null mice was reduced to half of that detected in the wild-type mice. No differences in the hepatic mRNA expression levels of tumor necrosis factor $\alpha$, interleukin $1 \beta$, or interleukin 6 were observed between the wild-type and Cygb-null mice (Figure 1d). Hepatic GSH level in both mouse lines decreased dramatically at $2 \mathrm{~h}$ after APAP challenge. After the maximum depletion, hepatic GSH level in Cygb-null mice recovered quickly, compared with that in wild-type mice (Figure 1e). Three of the tested five wild-type mice showed low hepatic GSH level at $6 \mathrm{~h}$ after APAP injection, compared with Cygb-null mice (Figure 1f). Taken together, these results suggest that CYGB deficiency restrained APAP-induced acute liver injury.

\section{Reduced Serum APAP Metabolites and Lower Hepatic $\mathrm{O}_{2}$ Levels in Cygb-Null Mice}

The hepatic expression of CYP2E1, an enzyme that generates NAPQI from APAP, was investigated in wild-type and Cygb-null mice. We confirmed previous reports indicating that hepatic CYP2E1 mRNA levels decreased in a 
a

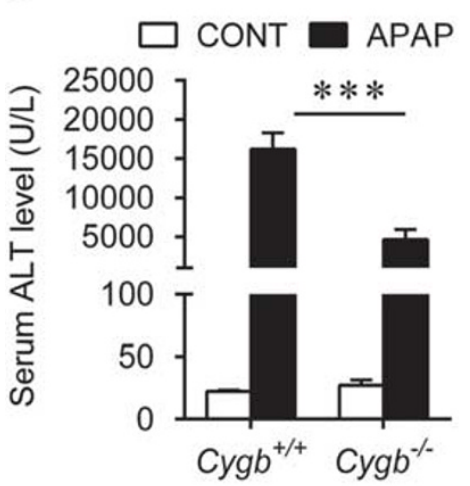

b

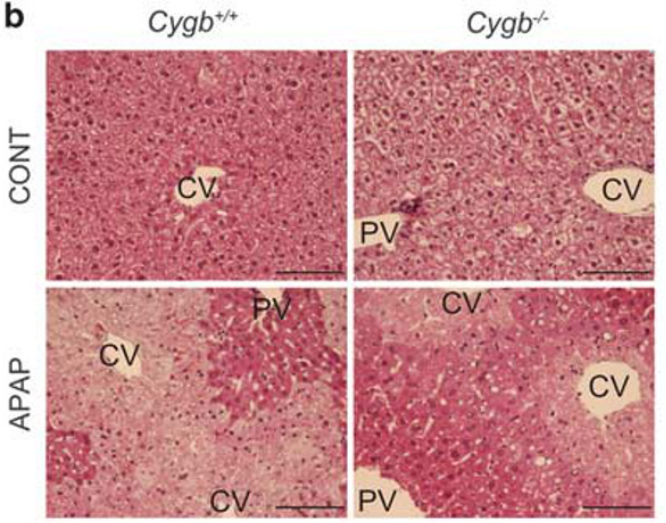

c

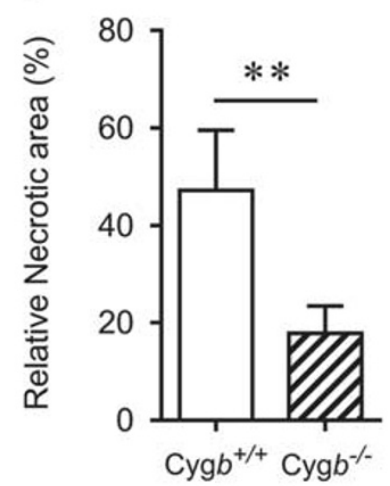

d

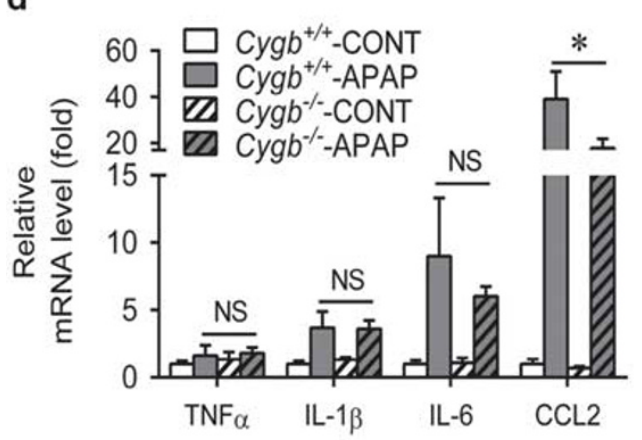

e

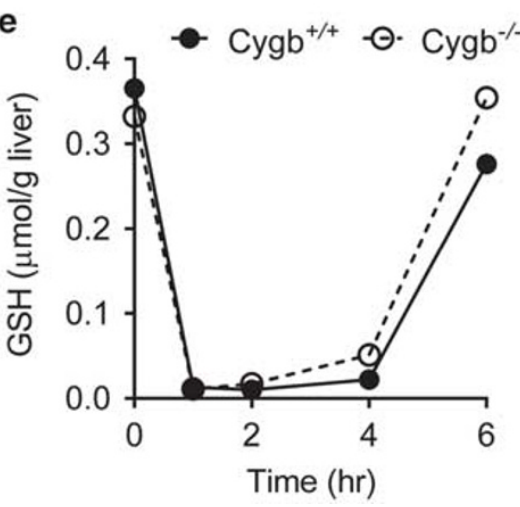

f

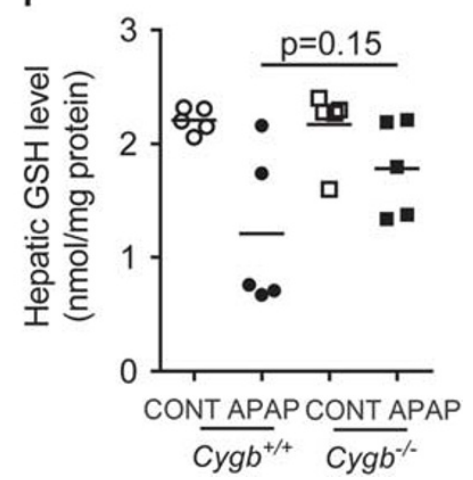

Figure 1 Analysis of $\mathrm{N}$-acetyl-p-aminophenol (APAP)-induced acute liver injury in wild-type and cytoglobin (Cygb)-null mice. (a) Serum alanine aminotransferase (ALT) levels. ALT activity was measured $6 \mathrm{~h}$ after saline (CONT) or $300 \mathrm{mg} / \mathrm{kg} \mathrm{APAP}$ administration. Each bar represents the mean value and s.d. ( $n=5-6)$. (b) Representative liver sections stained with hematoxylin and eosin ( $\times 400$, bar $=100 \mu \mathrm{m})$. CV, central vein. PV, portal vein. (c) Relative necrotic area was represented by the percentage ratio of necrotic area to liver area. Each bar represents the mean value and s.d. $(n=5)$. (d) Quantitative reverse transcription-PCR analysis of tumor necrosis factor- $\alpha$ (TNF- $\alpha$ ), interleukin (IL)-1 $\beta$, IL-6, and chemokine (C-C motif) ligand 2 (CCI2) mRNAs during APAP-induced liver injury. Each bar represents the mean value and s.d. $(n=5-6)$. (e) Time course of glutathione (GSH) levels in the liver $(n=2)$. (f) Hepatic GSH level at 6 h. Each bar represents the mean value $(n=5)$. Wild-type and $C y g b$-null mice are designated $C y g b^{+/+}$and $C y g b^{-\prime-}$, respectively. Significance was determined by a one-way ANOVA with Bonferroni's test. ${ }^{*} P<0.05$, ${ }^{* * *} P<0.001$; NS, not significant.

time-dependent manner after APAP injection in wild-type mice (Supplementary Figure S1C). ${ }^{18}$ No differences were noted in the CYP2E1 mRNA and protein levels between untreated wild-type and Cygb-null mice (Figures 2a and b). After APAP challenge, similar decreases in the CYP2E1 mRNA and protein levels were observed in the wild-type and Cygbnull mice. Furthermore, the hepatic microsomal CYP2E1 activity in vitro was assessed. The Michaelis-Menten kinetics curve for the CYP2E1 activity of Cygb-null mice were almost same to that for the wild-type mice. In addition, the $V_{\max }$ and the $K_{\mathrm{m}}$ were $0.325 \pm 0.10 \mathrm{nmol}$ per $\mathrm{mg}$ protein $/ \mathrm{min}$ and $17.48 \pm$ $9.85 \mu \mathrm{M}$ for the wild-type mice, and $0.338 \pm 0.03$ nmolper $\mathrm{mg}$ protein/min and $19.72 \pm 2.58 \mu \mathrm{M}$ for the $C y g b$-null mice, respectively. The differences between the wild-type and the Cygb-null mice were not observed (Figure 2c). However, the serum levels of CYP2E1-dependent APAP metabolites, ie, cysteinyl-APAP and $N$-acetyl-cysteinyl-APAP, which are derived from NAPQI (Figure 2d), were significantly decreased in the $C y g b$-null mice compared with the wild-type mice. In contrast, the APAP levels tended to be high in the Cygb-null mice and the levels of APAP-glucuronide, a CYP2E1-independent metabolite, remained unchanged (Figure 2e).

As $\mathrm{O}_{2}$ is required for the CYP2E1-mediated NAPQI generation, we hypothesized that the livers of the Cygb-null mice had low concentrations of $\mathrm{O}_{2}$ compared with the wild-type mice. To test this hypothesis, pimonidazole was used, which is frequently used to detect hypoxic regions in tissues. ${ }^{19}$ The resulting pimonidazole staining was limitedly positive around the central vein of the untreated livers. However, after treatment with APAP for $6 \mathrm{~h}$, the pimonidazole-positive areas expanded to zone 2 (intermediate area) in the livers of the $C y g b$-null mice, but not in the wild-type mice (Figure $3 a$ ). Although HIF-1 $\alpha$ protein was not detected (Figure $3 b$ ), the data may support that CYGB deficiency results in decreased hepatic $\mathrm{O}_{2}$ levels after APAP challenge.

\section{Attenuation of APAP-Induced Cell Death in Primary-Cultured Mouse Hepatocytes by Hypoxia}

Cultured primary mouse hepatocytes were challenged with 2.5-20 mM APAP under 5 or $20 \% \mathrm{O}_{2}$ for $16 \mathrm{~h}$. The APAP 
a

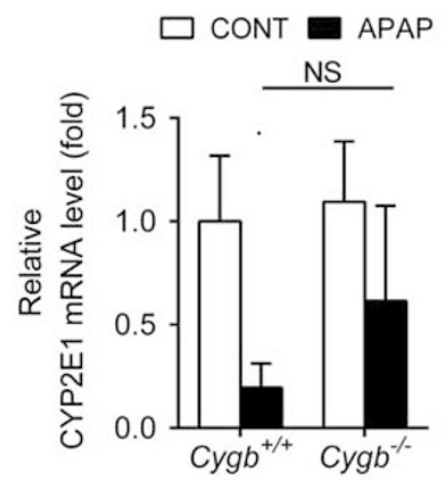

b

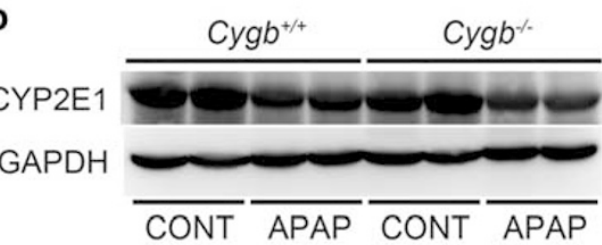

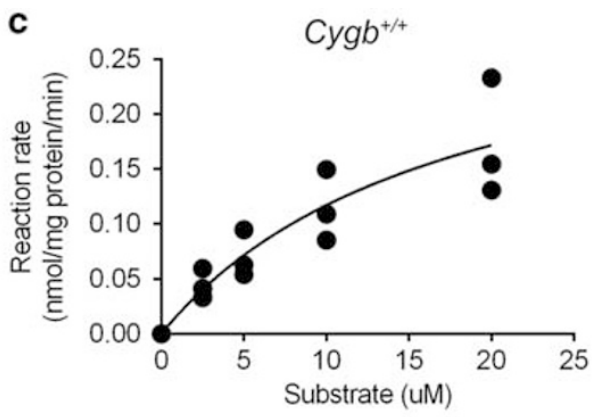

$\mathrm{Km}=17.48 \mathrm{uM}$,

Vmax $=0.325 \mathrm{nmol} / \mathrm{mg}$ protein $/ \mathrm{min}$

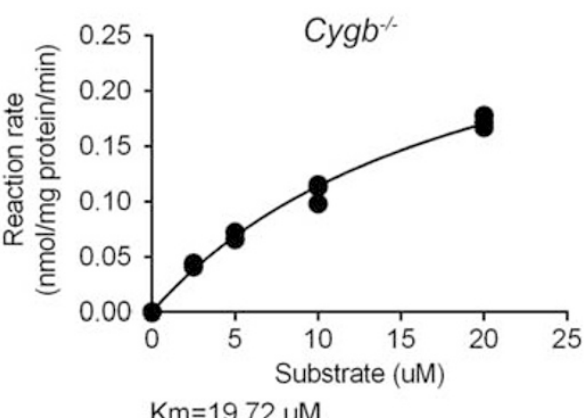

Vmax $=0.338 \mathrm{nmol} / \mathrm{mg}$ protein $/ \mathrm{min}$ d

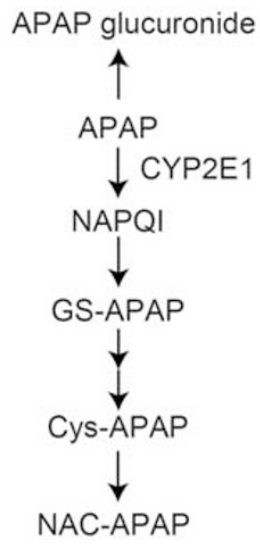

e

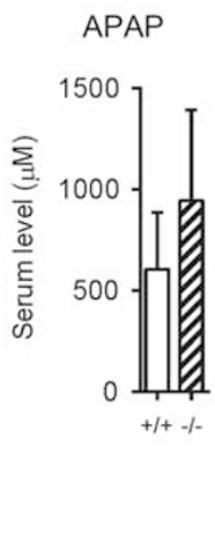

NAC-APAP

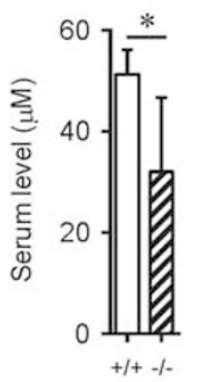

Cys-APAP

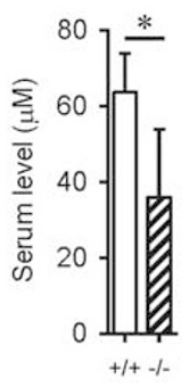

Figure 2 Cytochrome P450, family 2, subfamily E, polypeptide 1 (CYP2E1) expression and N-acetyl-p-aminophenol (APAP) metabolism in the liver. Quantitative reverse transcription-PCR (a) and western blot (b) analyses of CYP2E1 expression in the liver $6 \mathrm{~h}$ after APAP. Each bar represents the mean value and s.d. $(n=5-6)$. Significance was determined by a one-way ANOVA with Bonferroni's test (NS, not significant). (c) Major in vivo APAP metabolic pathways are indicated. GS-APAP, 3-gultathionyl-APAP; Cys-APAP, cysteinyl-APAP; NAC-APAP, N-acetyl-cysteinyl-APAP. (d) Serum levels of APAP and its major metabolites in $\mathrm{Cyg}^{+/+}$and $\mathrm{Cyg}^{-/-}$mice. Serum samples were collected $2 \mathrm{~h}$ after the administration of APAP, and APAP metabolites were measured by a liquid chromatography-mass spectrometry (LC-MS) analysis. The values are expressed as the relative abundance (mean and s.d., $n=5$ ). Significance was determined using an unpaired $t$-test $\left({ }^{*} P<0.05\right)$. (e) Hepatic microsomal CYP2E1 activity. The reaction rate was determined as described in Materials and Methods.

challenge induced cell death in a dose-dependent manner under $20 \% \mathrm{O}_{2}$. Notably, the hepatocyte damage was significantly greater under $20 \% \mathrm{O}_{2}$ than under $5 \% \mathrm{O}_{2}$ : when cells were treated with $20 \mathrm{mM}$ APAP, the hepatocyte death in the presence of 20 and $5 \% \mathrm{O}_{2}$ was 67.0 and $31.8 \%$, respectively (Figures $4 \mathrm{a}$ and $\mathrm{b}$ ). In accordance with these results, cell viability after exposure to APAP was lower in the presence of $20 \% \mathrm{O}_{2}$ than in the presence of $5 \% \mathrm{O}_{2}$ (Figure 4c). HIF- $1 \alpha$ protein was detected in the presence of $1 \% \mathrm{O}_{2}$, but not in the presence of 5 and $20 \% \mathrm{O}_{2}$ (Figure $4 \mathrm{~d}$ ). These results demonstrated that the $\mathrm{O}_{2}$ concentration influences the APAP toxicity in primary-cultured hepatocytes without HIF1 $\alpha$ activation. 


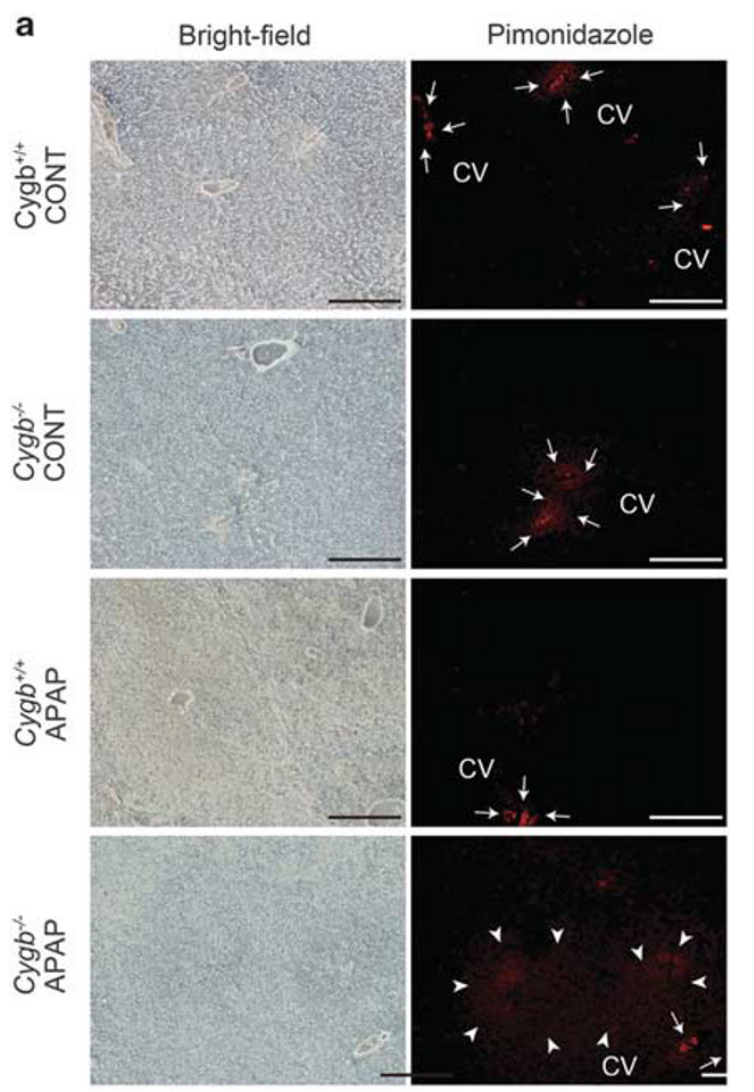

b

$\alpha-H I F 1 \alpha$

CBB

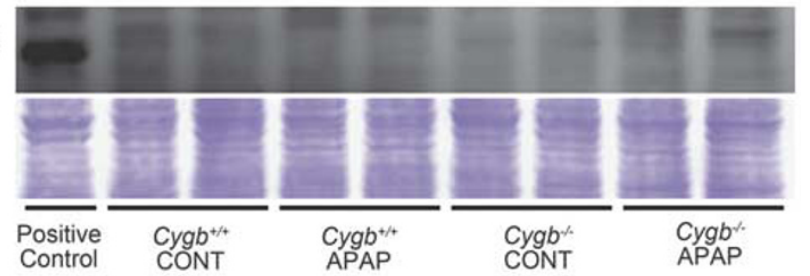

Figure 3 Determination of hypoxic conditions in mouse liver. (a) Pimonidazole staining in the livers of cytoglobin $(C y g b)^{+/+}($left $)$and $C y g b^{-/-}($right) mice $6 \mathrm{~h}$ after APAP treatment. Arrowheads indicate pimonidazole-positive areas. Arrows indicate the central vein $(\mathrm{CV})(\times 100, \mathrm{bar}=200 \mu \mathrm{m})$.

(b) Western blotting of hypoxia-inducible factor $1 \alpha$ (HIF-1 $\alpha$ ) protein. Coomassie brilliant blue (CBB) staining was used for the normalization.

\section{Deterioration of the Viability of Primary-Cultured Mouse Hepatocytes by Co-Culture With HSCs Under APAP Treatment}

Next, we studied whether the presence of HSCs influences the viability of mouse hepatocytes under conditions of APAP treatment. No difference in cell viability following APAP challenge was observed between wild-type and Cygb-null hepatocytes in monoculture under 5\% $\mathrm{O}_{2}$ (Figure 5a). However, hepatocytes co-cultured with wild-type HSCs showed significantly lower cell viability after APAP challenge compared with those co-cultured with Cygb-null HSCs: the cell viability values of hepatocytes in monoculture, those cocultured with wild-type HSCs, and those co-cultured with Cygb-null HSCs were $0.29,0.20$, and 0.32 , respectively (Figure 5b). In addition, hepatocytes showed a similar sensitivity to APAP when conditioned media from wild-type and Cygb-null HSCs were applied (Figure 5c). These results support the hypothesis that HSC-CYGB influences the behavior of hepatocytes, presumably through the $\mathrm{O}_{2}$ supply.

\section{Alleviation of $\mathrm{CCL}_{4}$-Induced, but Not LPS/D-GaIN- Induced, Acute Liver Injury By CYGB Deficiency}

To investigate whether the mitigation of hepatocyte damage is unique to APAP in Cygb-null mice, two other acute liver injury models, ie, $\mathrm{CCl}_{4}$-induced and LPS/D-GalN-induced liver damage, were employed. $\mathrm{CCl}_{4}$ is metabolized by CYP2E1, similar to APAP. In contrast, LPS-induced liver injury is generally associated with Toll-like receptor 4 signaling pathways and is independent of CYP activity. In the $\mathrm{CCl}_{4}$ model, the serum ALT levels were significantly higher in the wild-type mice $(14800 \pm 1500 \mathrm{U} / \mathrm{l})$ than in the Cygbnull mice $(4340 \pm 1030 \mathrm{U} / \mathrm{l})$, similar to the APAP model (Figure 6a). The histologic analysis revealed that centrilobular necrosis was more severe in the wild-type mice than in the Cygb-null mice (Figure 6b). In contrast, in the LPS/DGalN model the serum ALT levels of the wild-type mice did not differ from those of the Cygb-null mice (333 \pm 116 and $402 \pm 189 \mathrm{U} / \mathrm{l}$, respectively) (Figure 6c). A pathological analysis revealed the degeneration and necrosis of hepatocytes, the destruction of the hepatic architecture, and the presence of hemorrhage in both mouse strains (Figure 6d). These data support the hypothesis that CYGB deficiency decelerates the CYP2E1-mediated xenobiotic toxicity in the liver.

\section{DISCUSSION}

The current study demonstrated that APAP-induced liver injury was attenuated in Cygb-null mice compared with wildtype mice. $\mathrm{CCl}_{4}$-induced liver injury was also alleviated in 
a

$20 \% \mathrm{O}_{2}$ Bright-field
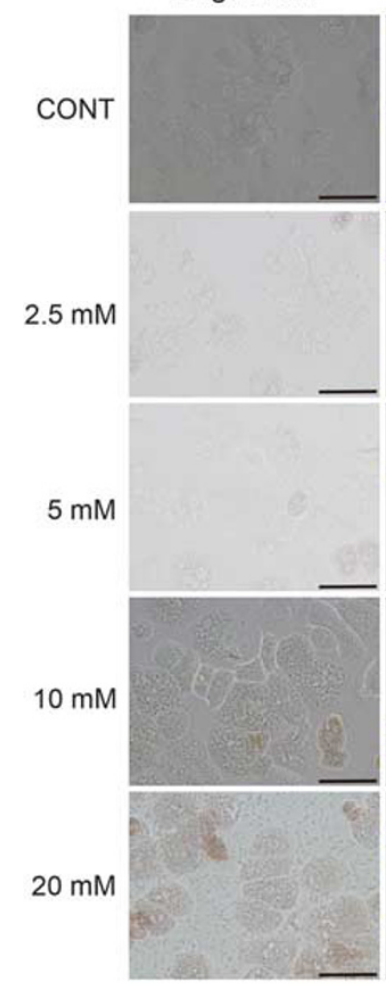

b

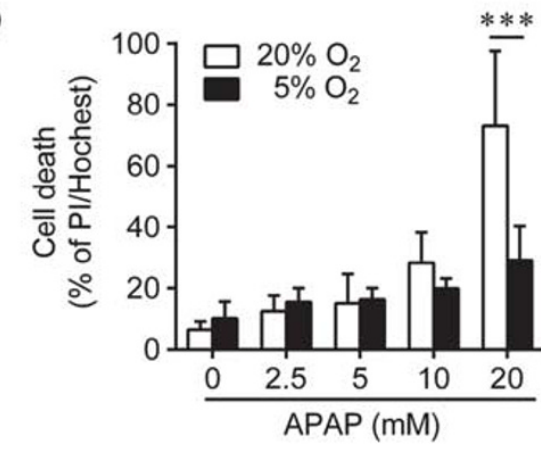

$5 \% \mathrm{O}_{2}$

Bright-field
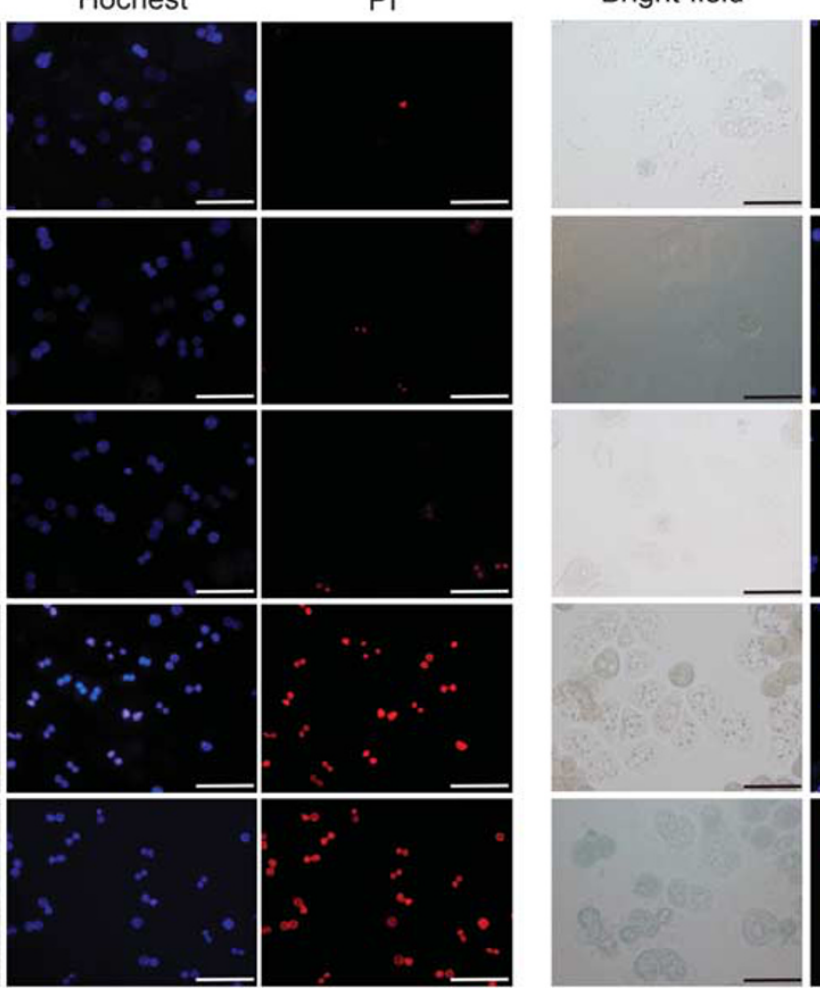

Hochest
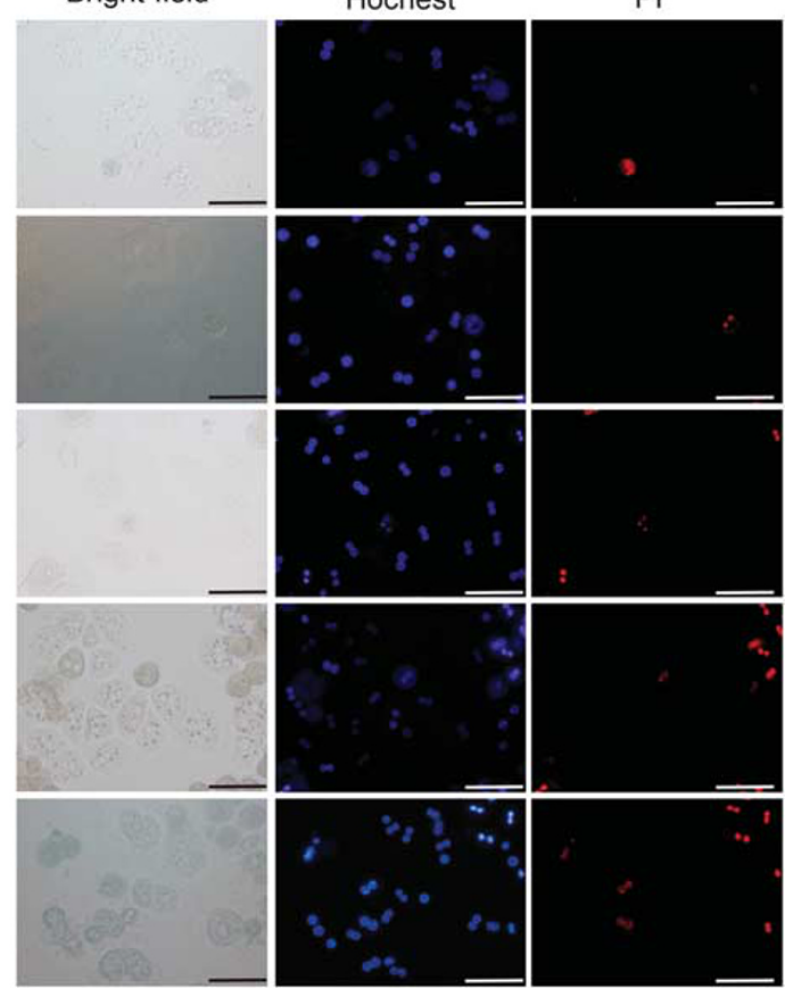

d
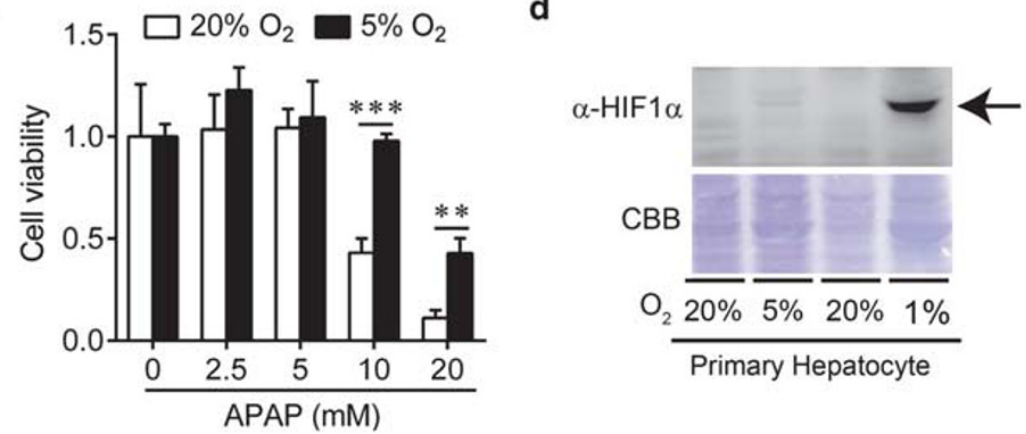

Figure 4 Influence of oxygen level on $\mathrm{N}$-acetyl-p-aminophenol (APAP)-induced hepatocyte injury in culture. (a) Representative images of APAP-induced hepatocyte damage. Cell death was determined using propidium iodide (PI) staining under 5 or $20 \%$ oxygen $\left(\mathrm{O}_{2}\right) 16 \mathrm{~h}$ after APAP administration. The concentrations of APAP were 0, 2.5, 5, 10, and $20 \mathrm{mM}$. Stained hepatocytes were observed under bright-field (left) and dark-field microscopy (center, Hoechst 33342; right, $\mathrm{Pl} ; \times 400$, bar $=50 \mu \mathrm{m}$ ). (b) Determination of cell death calculated as the ratio of PI-positive hepatocytes under 5 or $20 \% \mathrm{O}_{2}$. The values represent the percentage ratio of PI-positive hepatocytes to Hoechst-positive hepatocytes $(n=4-8)$. (c) Determination of cell viability by an MTT assay. Significance was determined by a two-way ANOVA with Bonferroni's test (b and $\mathbf{c}$ ). ${ }^{* *} P<0.01$, ${ }^{* * *} P<0.001$. (d) Western blotting of HIF-1 $\alpha$ protein under conditions of 1,5 , and $20 \% \mathrm{O}_{2}$. Arrow indicated HIF-1 $\alpha$ protein. Coomassie brilliant blue (CBB) staining was used for the normalization.

Cygb-null mice. APAP and $\mathrm{CCl}_{4}$ toxicity is mediated through the production of reactive metabolites by CYP2E1. The hepatic CYP2E1 expression levels and in the Cygb-null mice were similar to those of the wild-type mice; nevertheless, the serum levels of NAPQI derivatives, such as cysteinyl-APAP and $\mathrm{N}$-acetyl-cysteinyl-APAP, were lower in the Cygb-null mice than in the wild-type mice under APAP challenge. In addition, the serum APAP levels were elevated, albeit not significantly, in the Cygb-null mice compared with the wild-type mice. These results suggest that the generation of NAPQI is reduced in Cygb-null mice in the absence of changes in the hepatic CYP2E1 protein levels, and they support the theory that the enzymatic activity of CYP2E1 is attenuated in Cygb-null mice.

In this study, the hepatic microsomal CYP2E1 activity (under condition of disrupted sinusoidal microenvironment) in the Cygb-null mice was similar to that in the wild-type mice (Figure 2c). Considering that (1) CYPs are mono- 
a

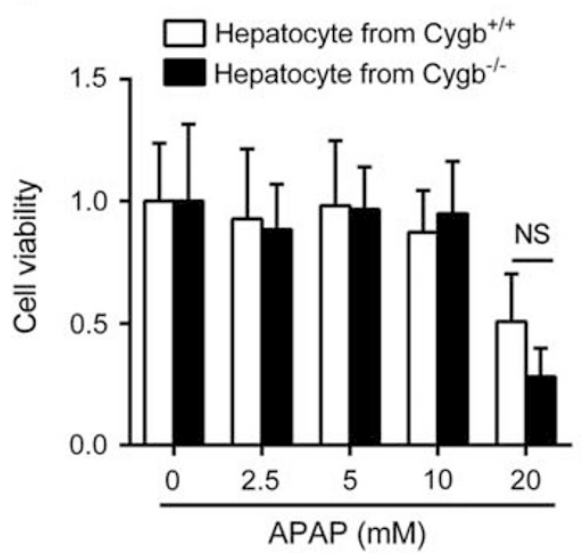

b

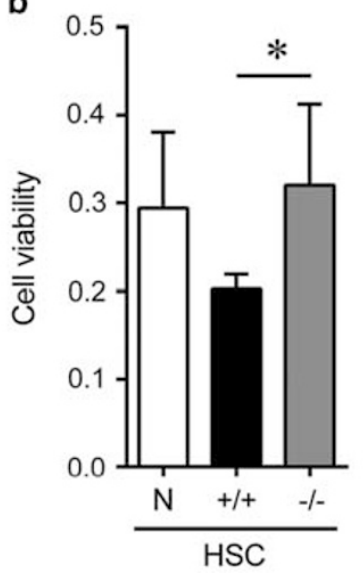

c

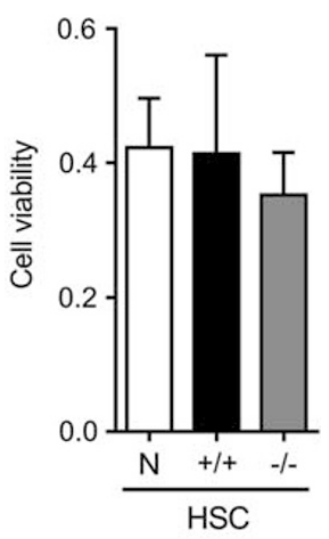

Figure 5 Presence of HSCs deteriorates the viability of mouse hepatocytes under conditions of APAP treatment. (a) Comparison of cell viability $16 \mathrm{~h}$ after APAP challenge between cytoglobin $(\mathrm{Cyg} b)^{+/+}$and $\mathrm{Cyg}^{-/-}$hepatocytes. (b) Effect of HSCs isolated from Cygb ${ }^{+/+}$or Cygb ${ }^{-/-}$mice on hepatocyte viability after $30 \mathrm{mM}$ APAP challenge for $16 \mathrm{~h}$ at low oxygen level $\left(5 \%\right.$ oxygen $\left.\left(\mathrm{O}_{2}\right)\right)$. White, black, and gray columns indicate without $\mathrm{HSC}$, with $\mathrm{Cygb}^{+/+} \mathrm{HSCs}$, and with Cygb $b^{-/-}$HSCs, respectively. (c) Effects of HSC-conditioned media on hepatocyte viability after 30 mM APAP challenge. Conditioned media were prepared as described in the Materials and Methods section. White, black, and gray columns indicate culture in the absence of conditioned medium, in the presence of conditioned medium from Cyg $b^{+/+} \mathrm{HSCs}$, and in the presence of conditioned medium from Cyg $b^{-/-}$ HSCs, respectively. Significance was determined by a two-way ANOVA with Bonferroni's test (a) and a one-way ANOVA with Bonferroni's test (b and c). ${ }^{*} P<0.05 ; \mathrm{NS}$, not significant.
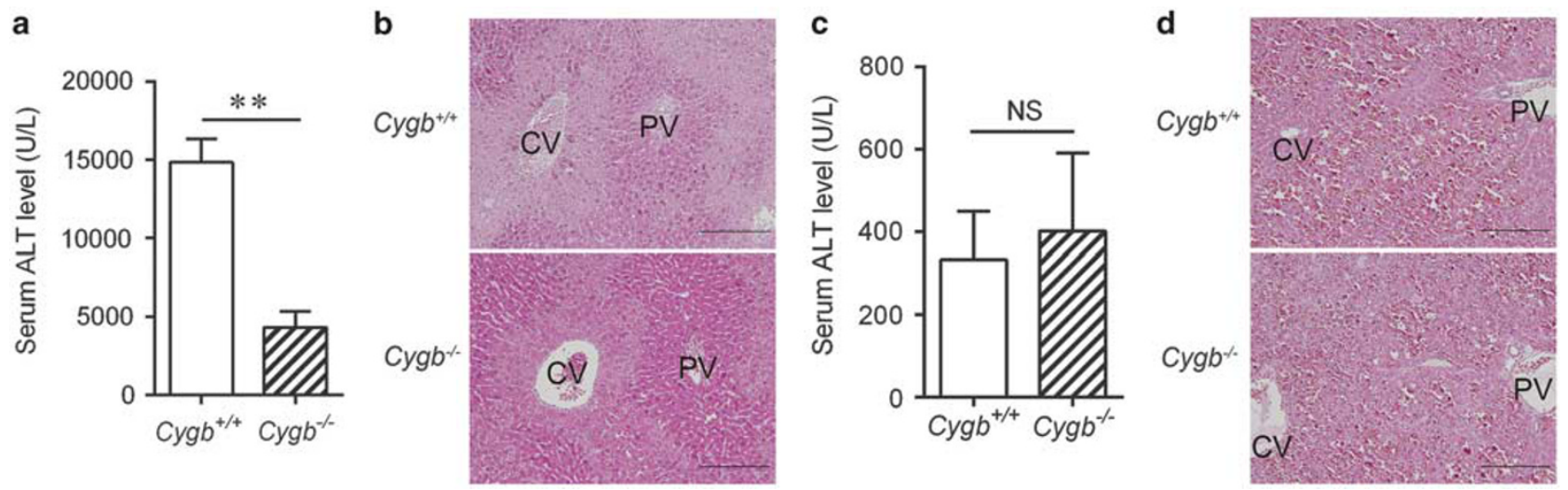

Figure 6 Carbon tetrachloride ( $\mathrm{CCl}_{4}$ )-induced and lipopolysaccharide (LPS)/D-galactosamine (D-GalN)-induced acute liver injury in mice. (a and b) Acute liver injury induced by $0.5 \mathrm{mg} / \mathrm{kg} \mathrm{CCl}_{4}$ administration. Serum alanine aminotransferase (ALT) activity (a) and liver histology (b; $\times 200$, bar $\left.=100 \mu \mathrm{m}\right) 24 \mathrm{~h}$ after $\mathrm{CCl}_{4}$ injection are shown (each, $n=3-4$ ). CV, central vein; PV, portal vein. (c and d) Acute liver injury induced by $700 \mathrm{mg} / \mathrm{kg}$ of $\mathrm{D}-\mathrm{GalN}$ and $5 \mu \mathrm{g} / \mathrm{kg}$ of LPS. Serum ALT activity (c) and liver histology $(\mathbf{d} ; \times 200$, bar $=100 \mu \mathrm{m}) 6 \mathrm{~h}$ after LPS/D-GalN injection are shown (each, $n=10)$. CV, central vein; $\mathrm{PV}$, portal vein. Significance was determined by an unpaired $t$-test $\left({ }^{* *} P<0.01 ; \mathrm{NS}\right.$, not significant).

oxygenases and (2) CYGB functions as an $\mathrm{O}_{2}$-binding protein, we concluded that the APAP-derived toxicity may differ with regard to hepatic $\mathrm{O}_{2}$ levels between $C y g b$-null and wild-type mice. In addition, pimonidazole-positive areas, indicating hypoxic conditions, were expanded only in the Cygb-null mice, although HIF $1 \alpha$ activation was not detected. As well as our observation, it has been reported that pimonidazole-positive cells are not always identical to HIF $1 \alpha$ activated cells. ${ }^{20,21}$ Thus, we considered that the Cygb-null mice exhibited low $\mathrm{O}_{2}$ tension in the liver, particularly after APAP challenge. Yan et al. ${ }^{5}$ showed that APAP-induced cell death in cultured mouse hepatocytes was attenuated in the presence of $10 \% \mathrm{O}_{2}$, which was accompanied by a decrease in mitochondrial oxidative stress. In the present study, we also observed that APAP-induced toxicity in mouse primary hepatocytes was alleviated even at lower $\mathrm{O}_{2}$ levels $\left(5 \% \mathrm{O}_{2}\right)$. Surprisingly, co-culture with wild-type HSCs, but not with Cygb-null HSCs, exacerbated the APAP-induced hepatocyte death under a $5 \% \mathrm{O}_{2}$ atmosphere. In addition, conditioned media from wild-type HSCs and from Cygb-null HSCs failed to influence the APAP-induced hepatocyte death, indicating that stable components in these conditioned media, such as proteins and lipids, were ineffective. Although the co-culture system used in this study can't be said to reproduce perfectly 
the sinusoidal environment in vivo, these results indicate that the HSC-CYGB could affect APAP-induced hepatocyte injury.

The $\mathrm{O}_{2}$ affinity of CYGB has been reported to be equivalent to that of myoglobin and neuroglobin, and is considerably higher than that of hemoglobin. ${ }^{6}$ This fact suggests that CYGB releases $\mathrm{O}_{2}$ at lower $\mathrm{O}_{2}$ tension compared with hemoglobin and, in combination, (1) HIF $1 \alpha$ protein was detected in the hepatocytes cultured with $1 \% \mathrm{O}_{2}$ but not in that with 20 and $5 \% \mathrm{O}_{2} ;(2)$ in fact, the wild-type HSCs, but not Cygb-null HSCs, can accelerate the APAP hepatocyte injury under $5 \% \mathrm{O}_{2}$; CYGB may be an early sensor for low oxygen level and supply $\mathrm{O}_{2}$ to neighboring cells before falling in hypoxia (activation of HIF). In other words, CYGB can have a role in $\mathrm{O}_{2}$ supply after the consumption of $\mathrm{O}_{2}$ derived from hemoglobin in vivo. When excess CYP2E1 metabolism causes a shortage of $\mathrm{O}_{2}$ in hepatocytes, the HSC-CYGB may supply $\mathrm{O}_{2}$ temporarily and locally to hepatocytes during the $\mathrm{O}_{2}$ insufficiency.

It has been reported that APAP damages the hepatic microvasculature, which precedes hepatocellular injury. In vivo microscopy indicated that this damage consists of endothelial cell swelling and the penetration of erythrocytes into the space of Disse. Significant decreases in the blood supply in hepatic sinusoids were observed 2 and $6 \mathrm{~h}$ after APAP administration. ${ }^{22}$ In contrast, when HSCs become activated, they undergo changes in the cytoskeletal machinery and gain the ability to contract. ${ }^{23}$ Some studies have demonstrated that activated HSCs contract in response to various agents. $^{24,25}$ Thus, activated HSCs are thought to be involved in the disturbance of hepatic microcirculation. ${ }^{26} \mathrm{~A}$ previous study reported that CYGB was able to suppress HSC activation. ${ }^{27}$ Considering all of these reports, the loss of CYGB may lead to the 'priming' of HSCs to the activated stage and can cause immediate sinusoidal constriction after APAP challenge due to HSC contraction. However, the hypothesis could be denied, because Cygb-null mice did not show attenuated LPS-induced liver injury.

In conclusion, the present study demonstrated in part that APAP-induced acute liver injury was attenuated in Cygb-null mice, presumably due to local low $\mathrm{O}_{2}$ level (but not hypoxia)

Figure 7 Putative mechanism by which hepatic stellate cell (HSC)expressed cytoglobin (CYGB) is involved in $\mathrm{N}$-acetyl-p-aminophenol (APAP)-induced liver injury. (a) The oxygen $\left(\mathrm{O}_{2}\right)$ concentration in the livers of Cygb-null mice was similar to that of wild-type mice under normal conditions. (b) At high APAP concentrations, APAP is metabolized by CYP2E1 and $\mathrm{O}_{2}$ is rapidly consumed. As a result, hepatocytes tend to be temporarily $\mathrm{O}_{2}$ deficient. $\mathrm{O}_{2}$ would be supplied from CYGB in HSCs located in the space of Disse in wild-type mice, but this is not the case in Cygb-null mice. (c) Thus, CYP2E1 efficiently generates $N$-acetyl-pbenzoquinone imine (NAPQI) from APAP in wild-type mice. In contrast, in Cygb-null mice NAPQI production is decreased because of impaired local $\mathrm{O}_{2}$ supply from $\mathrm{HSCs}$; thus, NAPQI-dependent hepatocyte injury is attenuated. Blue arrows indicate $\mathrm{O}_{2}$ transfer. Bold black arrows indicate the pathway by which APAP toxicity is mediated. Dashed line arrows indicate reduction. around hepatocytes closely adherent to HSCs (Figure 7). Although the molecular mechanism of $\mathrm{O}_{2}$ transfer from HSCs to hepatocytes remains uncharacterized, this study
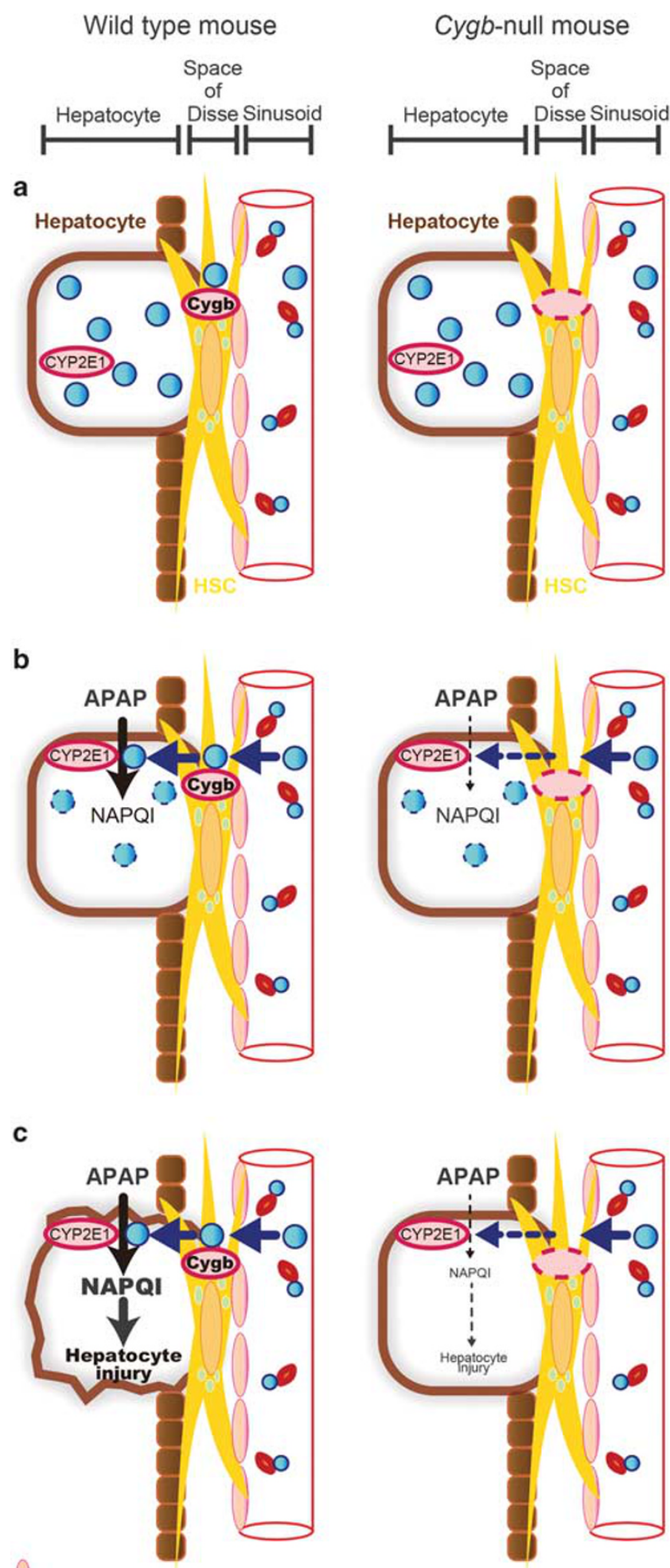

sinusoidal endothelial cell $\bigcirc$ oxygen Erythrocyte 
provides important evidence to further understand the molecular mechanism of drug-induced liver injury mediated by CYP2E1.

Supplementary Information accompanies the paper on the Laboratory Investigation website (http://www.laboratoryinvestigation.org)

\section{ACKNOWLEDGMENTS}

We thank Ms Chiho Kadono for animal care, and Dr Wenhao Cui, Dr Keiko Iwaisako, and Dr Kazuki Nakatani (Osaka City University) for their valuable discussion and comments on this study. This investigation was supported in part by a Grant-in-Aid for Scientific Research (B) from the Japan Society for the Promotion of Science (JSPS) through grant 25293177 (to NK) (20132016), the Mochida Memorial Foundation (to TM), and the National Cancer Institute Intramural Research Program (to FJG).

\section{AUTHOR CONTRIBUTIONS}

YT, TM, and NK conceived and designed the experiments. KWK and FJG quantified the APAP metabolites. LT provided technical assistance in the isolation of hepatocytes and $\mathrm{HSCS}$. KI and $\mathrm{KY}$ contributed critically to the design of the manuscript in terms of important intellectual content. YT, TM, and NK wrote the manuscript, with suggestions from all authors.

\section{DISCLOSURE/CONFLICT OF INTEREST}

The authors declare no conflict of interest.

1. Lieber CS. Cytochrome P-4502E1: its physiological and pathological role. Physiol Rev 1997;77:517-544.

2. Gonzalez FJ. The 2006 Bernard B. Brodie Award Lecture. Cyp2e1. Drug Metab Dispos 2007;35:1-8.

3. Gonzalez FJ, Kimura S. Study of P450 function using gene knockout and transgenic mice. Arch Biochem Biophys 2003;409:153-158.

4. Chen C, Krausz KW, Idle JR, et al. Identification of novel toxicity-associated metabolites by metabolomics and mass isotopomer analysis of acetaminophen metabolism in wild-type and Cyp2e1-null mice. J Biol Chem 2008;283:4543-4559.

5. Yan HM, Ramachandran A, Bajt ML, et al. The oxygen tension modulates acetaminophen-induced mitochondrial oxidant stress and cell injury in cultured hepatocytes. Toxicol Sci 2010;117:515-523.

6. Hankeln T, Ebner B, Fuchs $C$, et al. Neuroglobin and cytoglobin in search of their role in the vertebrate globin family. J Inorg Biochem 2005;99:110-119.

7. Tosqui $\mathrm{P}$, Colombo MF. Neuroglobin and cytoglobin: two new members of globin family. Rev Bras Hematol Hemoter 2011;33:307-311.

8. Avivi A, Gerlach F, Joel A, et al. Neuroglobin, cytoglobin, and myoglobin contribute to hypoxia adaptation of the subterranean mole rat Spalax. Proc Natl Acad Sci USA 2010;107:21570-21575.
9. Nakatani K, Okuyama H, Shimahara $\mathrm{Y}$, et al. Cytoglobin/STAP, its unique localization in splanchnic fibroblast-like cells and function in organ fibrogenesis. Lab Invest 2004;84:91-101.

10. Schmidt M, Gerlach F, Avivi A, et al. Cytoglobin is a respiratory protein in connective tissue and neurons, which is up-regulated by hypoxia. J Biol Chem 2004;279:8063-8069.

11. Oleksiewicz U, Liloglou T, Field JK, et al. Cytoglobin: biochemical, functional and clinical perspective of the newest member of the globin family. Cell Mol Life Sci 2011;68:3869-3883.

12. Kawada N, Kristensen DB, Asahina K, et al. Characterization of a stellate cell activation-associated protein (STAP) with peroxidase activity found in rat hepatic stellate cells. J Biol Chem 2001;276:25318-25323.

13. Mabuchi A, Mullaney I, Sheard PW, et al. Role of hepatic stellate cell/ hepatocyte interaction and activation of hepatic stellate cells in the early phase of liver regeneration in the rat. J Hepatol 2004;40:910-916.

14. Yin C, Evason KJ, Asahina K, et al. Hepatic stellate cells in liver development, regeneration, and cancer. J Clin Invest 2013;123:1902-1910.

15. Thuy le $\Pi$, Morita $\mathrm{T}$, Yoshida $\mathrm{K}$, et al. Promotion of liver and lung tumorigenesis in DEN-treated cytoglobin-deficient mice. Am J Pathol 2011;179:1050-1060.

16. Iwaisako K, Hatano E, Taura K, et al. Loss of Sept4 exacerbates liver fibrosis through the dysregulation of hepatic stellate cells. J Hepatol 2008:49:768-778.

17. da Rosa EJ, da Silva MH, Carvalho NR, et al. Reduction of acute hepatic damage induced by acetaminophen after treatment with diphenyl diselenide in mice. Toxicol Pathol 2012;40:605-613.

18. Ishibe $T$, Kimura A, Ishida $Y$, et al. Reduced acetaminophen-induced liver injury in mice by genetic disruption of IL-1 receptor antagonist. Lab Invest 2009;89:68-79.

19. Samoszuk MK, Walter J, Mechetner E. Improved immunohistochemical method for detecting hypoxia gradients in mouse tissues and tumors. J Histochem Cytochem 2004;52:837-839.

20. Harada $\mathrm{H}$, Inoue $\mathrm{M}$, Itasaka $\mathrm{S}$, et al. Corrigendum: Cancer cells that survive radiation therapy acquire HIF-1 activity and translocate toward tumour blood vessels. Nat Commun 2013;4:2314.

21. Tanaka H, Yamamoto M, Hashimoto N, et al. Hypoxia-independent overexpression of hypoxia-inducible factor 1 alpha as an early change in mouse hepatocarcinogenesis. Cancer Res 2006;66:11263-11270.

22. Hinson JA, Roberts DW, James LP. Mechanisms of acetaminopheninduced liver necrosis. Handb Exp Pharmacol 2010369-405.

23. Jiang JX, Torok NJ. Liver Injury and the Activation of the Hepatic Myofibroblasts. Curr Pathobiol Rep 2013;1:215-223.

24. Friedman SL. Hepatic stellate cells: protean, multifunctional, and enigmatic cells of the liver. Physiol Rev 2008;88:125-172.

25. Reynaert $\mathrm{H}$, Thompson MG, Thomas $\mathrm{T}$, et al. Hepatic stellate cells: role in microcirculation and pathophysiology of portal hypertension. Gut 2002;50:571-581.

26. Vollmar B, Menger MD. The hepatic microcirculation: mechanistic contributions and therapeutic targets in liver injury and repair. Physiol Rev 2009:89:1269-1339.

27. Cui W, Wang M, Maegawa $\mathrm{H}$, et al. Inhibition of the activation of hepatic stellate cells by arundic acid via the induction of cytoglobin. Biochem Biophys Res Commun 2012;425:642-648. 